\title{
A Stability Result for the Solutions of a Certain System of Fourth-Order Delay Differential Equation
}

\author{
A. M. A. Abou-El-Ela, ${ }^{1}$ A. I. Sadek, ${ }^{1}$ A. M. Mahmoud, ${ }^{2}$ and R. O. A. Taie ${ }^{1}$ \\ ${ }^{1}$ Department of Mathematics, Faculty of Science, Assiut University, Assiut 71516, Egypt \\ ${ }^{2}$ Department of Mathematics, Faculty of Science, Assiut University, New Valley Branch, New Valley, El-khargah 72111, Egypt
}

Correspondence should be addressed to R. O. A. Taie; rasha_omath@yahoo.com

Received 25 September 2014; Revised 11 February 2015; Accepted 13 February 2015

Academic Editor: Davood D. Ganji

Copyright (C) 2015 A. M. A. Abou-El-Ela et al. This is an open access article distributed under the Creative Commons Attribution License, which permits unrestricted use, distribution, and reproduction in any medium, provided the original work is properly cited.

The main purpose of this work is to give sufficient conditions for the uniform stability of the zero solution of a certain fourth-order vector delay differential equation of the following form: $X^{(4)}+F(\dot{X}, \ddot{X}) \ddot{X}+\Phi(\ddot{X})+G(\dot{X}(t-r))+H(X(t-r))=0$. By constructing a Lyapunov functional, we obtained the result of stability.

\section{Introduction}

As is well known, the stability is a very important problem in the theory and applications of delay differential equations. Therefore, in the literature, some methods have been developed to obtain information on the stability behaviour of the delay differential equations when there is no analytical expression for the solutions. One of these methods is known as Lyapunov's second method; since Lyapunov [1] proposed his famous second method on the stability of motion, the problems related to the investigation of stability of solutions of certain second-, third-, and fourth-order linear and nonlinear, scalar, and vector differential equations have been given great attention in the past five decades due to the importance of the subject.

During this period, stability of solutions for various higher-order linear and nonlinear differential equations has been extensively studied and many results have been obtained in the literature (see, e.g., Krasovskii [2], Yoshizawa [3], Reissig et al. [4], Abou-El-Ela and Sadek [5-7], Bereketoglu and Kart [8], Sadek [9], Tunç [10-13], Abou-El-Ela et al. [14], and the references cited in those works), among which the results performed on asymptotic stability properties of linear and nonlinear scalar and vector differential equations of fourth-order can briefly be summarized as follows.
First in 1990 Abou-El-Ela and Sadek [5] found sufficient conditions for the asymptotic stability of the zero solution of the scalar nonlinear differential equation of the form

$$
x^{(4)}+f_{1}(\dot{x}, \ddot{x}) \dddot{x}+f_{2}(\dot{x}, \ddot{x})+f_{3}(x, \dot{x})+f_{4}(x)=0 .
$$

Later in 2004 Sadek [9] determined sufficient conditions, under which all solutions of the nonhomogeneous vector differential equation

$$
\begin{aligned}
X^{(4)} & +F(\dot{X}, \ddot{X}) \ddot{X}+\Phi(\ddot{X})+G(\dot{X})+A_{4} X \\
& =P(t, X, \dot{X}, \ddot{X}, \dddot{X})
\end{aligned}
$$

tend to zero as $t \rightarrow \infty$.

Recently in 2012 Abou-El-Ela et al. [14] investigated sufficient conditions for the uniform stability of the zero solution of the real fourth-order vector delay differential equation

$$
X^{(4)}+A \dddot{X}+\Phi(\ddot{X})+G(\dot{X})+H(X(t-r))=0 .
$$

In the present paper, we are concerned with the uniform stability of the zero solution $X=0$ of real nonlinear 
autonomous vector delay differential equation of the fourthorder

$$
\begin{aligned}
& X^{(4)}+F(\dot{X}, \ddot{X}) \dddot{X}+\Phi(\ddot{X})+G(\dot{X}(t-r)) \\
& +H(X(t-r))=0,
\end{aligned}
$$

where $X \in R^{n}$; $F$ is an $n \times n$-symmetric matrix; $\Phi, G$, and $H$ are $n$-vector continuous functions; $\Phi(0)=G(0)=H(0)=0$; and $r$ is a bounded delay and positive constant.

Equation (4) represents a system of real fourth-order differential equation with delay

$$
\begin{aligned}
x_{i}^{(4)} & +\sum_{k=1}^{n} f_{i k}\left(\dot{x}_{1}, \ldots, \dot{x}_{n} ; \ddot{x}_{1}, \ldots, \ddot{x}_{n}\right) \dddot{x}_{k}+\phi_{i}\left(\ddot{x}_{1}, \ldots, \ddot{x}_{n}\right) \\
& +g_{i}\left(\dot{x}_{1}(t-r), \ldots, \dot{x}_{n}(t-r)\right) \\
& +h_{i}\left(x_{1}(t-r), \ldots, x_{n}(t-r)\right)=0, \quad(i=1,2, \ldots, n) .
\end{aligned}
$$

The Jacobian matrices $J(F(Y, Z) Y \mid Z), J(F(Y, Z) Z \mid Z)$, $J(F(Y, Z) Y \mid Y), J(F(Y, Z) Z \mid Y), J_{\Phi}(Z), J_{G}(Y)$, and $J_{H}(X)$ are given by

$$
\begin{aligned}
& J(F(Y, Z) Y \mid Z)=\left(\frac{\partial}{\partial z_{j}} \sum_{k=1}^{n} f_{i k} y_{k}\right)=\left(\sum_{k=1}^{n} \frac{\partial f_{i k}}{\partial z_{j}} y_{k}\right), \\
& J(F(Y, Z) Z \mid Z)=\left(\frac{\partial}{\partial z_{j}} \sum_{k=1}^{n} f_{i k} z_{k}\right) \\
& =F(Y, Z)+\left(\sum_{k=1}^{n} \frac{\partial f_{i k}}{\partial z_{j}} z_{k}\right) \\
& J(F(Y, Z) Y \mid Y)=\left(\frac{\partial}{\partial y_{j}} \sum_{k=1}^{n} f_{i k} y_{k}\right) \\
& =F(Y, Z)+\left(\sum_{k=1}^{n} \frac{\partial f_{i k}}{\partial y_{j}} y_{k}\right) \\
& J(F(Y, Z) Z \mid Y)=\left(\frac{\partial}{\partial y_{j}} \sum_{k=1}^{n} f_{i k} z_{k}\right)=\left(\sum_{k=1}^{n} \frac{\partial f_{i k}}{\partial y_{j}} z_{k}\right) \text {, } \\
& J_{\Phi}(Z)=\left(\frac{\partial \phi_{i}}{\partial z_{j}}\right), \quad J_{G}(Y)=\left(\frac{\partial g_{i}}{\partial y_{j}}\right), \\
& J_{H}(X)=\left(\frac{\partial h_{i}}{\partial x_{j}}\right)
\end{aligned}
$$

where $(i, j=1,2, \ldots, n),\left(x_{1}, \ldots, x_{n}\right),\left(y_{1}, \ldots, y_{n}\right),\left(z_{1}, \ldots\right.$, $\left.z_{n}\right),\left(f_{i k}\right),\left(\phi_{1}, \ldots, \phi_{n}\right),\left(g_{1}, \ldots, g_{n}\right)$, and $\left(h_{1}, \ldots, h_{n}\right)$ represent $X, Y, Z, F, \Phi, G$, and $H$, respectively. It will also be assumed as basic throughout the paper that the Jacobian matrices $J(F(Y, Z) Y \mid Z), J(F(Y, Z) Z \mid Z), J(F(Y, Z) Y \mid Y), J(F(Y$, $Z) Z \mid Y), J_{\Phi}(Z), J_{G}(Y)$, and $J_{H}(X)$ exist and are continuous. The symbol $\langle X, Y\rangle$ will be used to denote the usual scalar product in $R^{n}$ for any $X, Y$ in $R^{n}$; that is, $\langle X, Y\rangle=\sum_{i=1}^{n} x_{i} y_{i}$; thus $\langle X, X\rangle=\|X\|^{2}$. It is well known that the real symmetric matrix $A=\left(a_{i j}\right),(i, j=1,2, \ldots, n)$ is said to be positivedefinite, if and only if the quadratic form $X^{T} A X$ is positivedefinite, where $X \in R^{n}$ and $X^{T}$ denotes the transpose of $X$.

\section{Main Result}

In order to reach the main result of this paper, we will give some basic information to the stability criteria for a general autonomous delay differential system. We consider

$$
\dot{\bar{x}}=\bar{f}\left(\bar{x}_{t}\right), \quad \bar{x}_{t}(s)=\bar{x}(t+s), \quad-h \leq s \leq 0, t \geq 0,
$$

where $\bar{f}: \mathscr{C}_{H} \rightarrow R^{n}$ is a continuous mapping, $\bar{f}(0)=0$, $\mathscr{C}_{H}:=\left\{\phi \in \mathscr{C}\left([-h, 0], R^{n}\right):\|\phi\| \leq H\right\}$, and for $H_{1}<H$, there exists an $L\left(H_{1}\right)>0$, with $|\bar{f}(\phi)| \leq L\left(H_{1}\right)$ when $\|\phi\|<H_{1}$.

Theorem 1 (see [15]). Let $V(\phi): \mathscr{C}_{H} \rightarrow R$ be a continuous functional satisfying a local Lipschitz condition, $V(0)=0$, such that

(i) $W_{1}(|\phi(0)|) \leq V(\phi) \leq W_{2}(\|\phi\|)$, where $W_{1}, W_{2}$ are wedges;

(ii) $\dot{V}_{(7)}(\phi) \leq 0$, for $\phi \in \mathscr{C}_{H}$.

Then the zero solution of (7) is uniformly stable. (4).

The following theorem will be our main stability result for

Theorem 2. In addition to the essential assumptions imposed on the functions $F, \Phi, G$, and $H$, suppose the existence of arbitrary positive constants $\alpha_{1}, \alpha_{2}, \alpha_{3}, \alpha_{4}, \alpha_{1}^{\prime}$, and $\alpha_{4}^{\prime}$. Suppose also for $i=1,2, \ldots, n$ the following conditions are satisfied.

(i) $F(Y, Z), J(F(Y, Z) Y \mid Z)$, and $J(F(Y, Z) Z \mid Z)$ are symmetric; $\alpha_{1}^{\prime} \geq \lambda_{i}(F(Y, Z)) \geq \alpha_{1}>0$, for all $Y, Z \in$ $R^{n}$.

(ii) $G(0)=0, J_{G}(Y)$ is symmetric and $\lambda_{i}\left(\int_{0}^{1} J_{G}(\sigma Y) d \sigma\right) \geq$ $\alpha_{3} \alpha_{4}^{2} / \alpha_{4}^{\prime 2}$, for all $Y \in R^{n}$.

(iii) There is a finite constant $\Delta>0$ such that

$\left\{\alpha_{1} \alpha_{2}-\left\|J_{G}(Y)\right\|\right\} \alpha_{3} \alpha_{4}-\alpha_{1} \alpha_{4}^{\prime 2}\left\|\int_{0}^{1} F(Y, \sigma Z) d \sigma\right\| \geq \Delta$,

for all $Y, Z \in R^{n}$.

(iv) One has $0 \leq \lambda_{i}\left(J_{G}(Y)-\int_{0}^{1} J_{G}(\sigma Y) d \sigma\right) \leq \delta_{1}<2 \Delta /$ $\alpha_{1} \alpha_{3}^{2}$, for all $Y \in R^{n}$.

(v) One has $\lambda_{i}\left(\int_{0}^{1} F(Y, \sigma Z) d \sigma-F(Y, Z)\right) \leq \delta_{2}<2 \Delta /$ $\alpha_{1}^{2} \alpha_{3} \alpha_{4}$, for all $Y, Z \in R^{n}$.

(vi) $J(F(Y, Z) Y \mid Y)-F(Y, Z)$ and $J(F(Y, Z) Z \mid Y)$ are negative-definite.

(vii) Also $H(0)=0, J_{H}(X)$ is symmetric, and $\lambda_{i}\left(\int_{0}^{1} J_{H}(\sigma X) d \sigma\right) \geq \alpha_{4}^{\prime}$, for all $X \in R^{n}$.

(viii) $J_{H}(X)$ commutes with $J_{H}\left(X^{\prime}\right)$, for all $X, X^{\prime} \in R^{n}$ and $0 \leq \lambda_{i}\left(\alpha_{4} I-J_{H}(X)\right) \leq \varepsilon D_{0} \alpha_{1}^{2}$, for all $X \in R^{n}$, and $D_{0}:=\alpha_{1} \alpha_{2}+\alpha_{2} \alpha_{3} \alpha_{4} \alpha_{4}^{\prime-2}$. 
(ix) Also $\Phi(0)=0, J_{\Phi}(Z)$ is symmetric, and $0 \leq$ $\lambda_{i}\left(\int_{0}^{1} J_{\Phi}(\sigma Z) d \sigma-\alpha_{2} I\right) \leq \varepsilon_{0} \alpha_{3}^{3} \alpha_{4}^{2} / \alpha_{4}^{\prime 4}$, for all $Z \in R^{n}$, where $\varepsilon_{0}$ is a positive constant such that

$$
\begin{array}{r}
\varepsilon_{0}<\varepsilon=\min \left\{\frac{1}{\alpha_{1}}, \frac{\alpha_{4}^{\prime 2}}{\alpha_{3} \alpha_{4}}, \frac{\Delta}{4 \alpha_{1} \alpha_{3} \alpha_{4} D_{0}},\right. \\
\frac{\alpha_{3} \alpha_{4}}{4 \alpha_{4}^{\prime 2} D_{0}}\left(\frac{2 \alpha_{4}^{\prime 2} \Delta}{\alpha_{1} \alpha_{3}^{2} \alpha_{4}^{2}}-\delta_{1}\right), \\
\left.\frac{\alpha_{1}}{4 D_{0}}\left(\frac{2 \Delta}{\alpha_{1}^{2} \alpha_{3} \alpha_{4}}-\delta_{2}\right)\right\} .
\end{array}
$$

Then the zero solution of (4) is uniformly stable, provided that

$$
\begin{aligned}
r<\min [ & \frac{\varepsilon}{d_{1} \sqrt{n}\left(\alpha_{4}+\alpha_{1} \alpha_{2}\right)}, \\
& \frac{\Delta}{2 \alpha_{1} \alpha_{3} \alpha_{4} \sqrt{n}\left\{\alpha_{4}+\alpha_{1} \alpha_{2}\left(d_{1}+d_{2}+2\right)\right\}}, \\
& \left.\frac{\left(\left(\alpha_{4}^{2} / \alpha_{4}^{\prime 2}\right) \varepsilon-\varepsilon_{0}\right) \alpha_{3}}{\alpha_{4} \sqrt{n}\left(d_{1}+2 d_{2}+1\right)+\alpha_{1} \alpha_{2} d_{2} \sqrt{n}}\right],
\end{aligned}
$$

where

$$
d_{1}=\varepsilon+\frac{1}{\alpha_{1}}, \quad d_{2}=\varepsilon+\frac{\alpha_{4}^{\prime 2}}{\alpha_{3} \alpha_{4}} .
$$

The following two lemmas are important for proving Theorem 2 .

Lemma 3. Let $A$ be a real symmetric $n \times n$-matrix and

$$
a^{\prime} \geq \lambda_{i}(A) \geq a>0 \quad(i=1,2, \ldots, n),
$$

where $a^{\prime}$, a are constants. Then

$$
\begin{gathered}
a^{\prime}\langle X, X\rangle \geq\langle A X, X\rangle \geq a\langle X, X\rangle, \\
a^{\prime 2}\langle X, X\rangle \geq\langle A X, A X\rangle \geq a^{2}\langle X, X\rangle .
\end{gathered}
$$

For a proof of the above lemma, see Bellman [16].

Lemma 4. Assume that $\dot{X}=Y, \dot{Y}=Z$, and $\dot{Z}=W$. Then

(1) $(d / d t) \int_{0}^{1}\langle H(\sigma X), X\rangle d \sigma=\langle H(X), Y\rangle$;

(2) $(d / d t) \int_{0}^{1}\langle G(\sigma Y), Y\rangle d \sigma=\langle G(Y), Z\rangle$;

(3) $(d / d t) \int_{0}^{1}\langle\Phi(\sigma Z), Z\rangle d \sigma=\langle\Phi(Z), W\rangle$;

(4) $(d / d t) \int_{0}^{1}\langle\sigma F(Y, \sigma Z) Z, Z\rangle d \sigma \leqq\langle F(Y, Z) Z, W\rangle$;

(5) $(d / d t) \int_{0}^{1}\langle F(Y, \sigma Z) Z, Y\rangle d \sigma \leqq\langle F(Y, Z) Y, W\rangle+$ $\left\|\int_{0}^{1} F(Y, \sigma Z) d \sigma\right\|\langle Z, Z\rangle$.
Proof. The proof is as follows:

(1) $\frac{d}{d t} \int_{0}^{1}\langle H(\sigma X), X\rangle d \sigma$

$$
\begin{aligned}
= & \int_{0}^{1} \sigma\left\langle J_{H}(\sigma X) Y, X\right\rangle d \sigma \\
& +\int_{0}^{1}\langle H(\sigma X), Y\rangle d \sigma \\
= & \int_{0}^{1} \sigma\left\langle J_{H}(\sigma X) X, Y\right\rangle d \sigma+\int_{0}^{1}\langle H(\sigma X), Y\rangle d \sigma \\
= & \int_{0}^{1} \sigma \frac{\partial}{\partial \sigma}\langle H(\sigma X), Y\rangle d \sigma+\int_{0}^{1}\langle H(\sigma X), Y\rangle d \sigma \\
= & \left.\sigma\langle H(\sigma X), Y\rangle\right|_{0} ^{1}=\langle H(X), Y\rangle .
\end{aligned}
$$

The proofs of (2) and (3) are similar to that of (1):

(4) $\frac{d}{d t} \int_{0}^{1}\langle\sigma F(Y, \sigma Z) Z, Z\rangle d \sigma$

$$
=\int_{0}^{1}\langle\sigma F(Y, \sigma Z) Z, W\rangle d \sigma
$$

$$
+\int_{0}^{1}\langle J(F(Y, \sigma Z) \sigma Z \mid Y) Z, Z\rangle d \sigma
$$$$
+\int_{0}^{1} \sigma\langle J(F(Y, \sigma Z) \sigma Z \mid \sigma Z) W, Z\rangle d \sigma
$$

$$
\begin{aligned}
& \leqq \int_{0}^{1}\langle\sigma F(Y, \sigma Z) Z, W\rangle d \sigma \\
& \quad+\int_{0}^{1} \sigma\langle J(F(Y, \sigma Z) \sigma Z \mid \sigma Z) Z, W\rangle d \sigma,
\end{aligned}
$$

since $J(F Z \mid Y)$ is negative-definite from assumption (vi) and $J(F Z \mid Z)$ is symmetric from assumption (i). Then

$$
\begin{aligned}
& \frac{d}{d t} \int_{0}^{1}\langle\sigma F(Y, \sigma Z) Z, Z\rangle d \sigma \\
& \leqq \int_{0}^{1}\langle\sigma F(Y, \sigma Z) Z, W\rangle d \sigma \\
&+\int_{0}^{1} \sigma \frac{\partial}{\partial \sigma}\langle\sigma F(Y, \sigma Z) Z, W\rangle d \sigma \\
&=\left.\sigma\langle\sigma F(Y, \sigma Z) Z, W\rangle\right|_{0} ^{1} \\
&=\langle F(Y, Z) Z, W\rangle,
\end{aligned}
$$


(5) $\frac{d}{d t} \int_{0}^{1}\langle F(Y, \sigma Z) Z, Y\rangle d \sigma$

$$
\begin{aligned}
= & \frac{d}{d t} \int_{0}^{1}\langle F(Y, \sigma Z) Y, Z\rangle d \sigma \\
= & \frac{d}{d t} \int_{0}^{1}\langle F(Y, \sigma Z) Y, W\rangle d \sigma \\
& +\int_{0}^{1}\langle J(F(Y, \sigma Z) Y \mid Y) Z, Z\rangle d \sigma \\
& +\int_{0}^{1}\langle\sigma J(F(Y, \sigma Z) Y \mid \sigma Z) W, Z\rangle d \sigma .
\end{aligned}
$$

Since $J(F Y \mid Z)$ is negative-definite from assumption (i), we have

$$
\begin{aligned}
\int_{0}^{1} & \langle\sigma J(F(Y, \sigma Z) Y \mid \sigma Z) W, Z\rangle d \sigma \\
& =\int_{0}^{1}\langle\sigma J(F(Y, \sigma Z) Y \mid \sigma Z) Z, W\rangle d \sigma \\
& =\int_{0}^{1} \sigma \frac{\partial}{\partial \sigma}\langle F(Y, \sigma Z) Y, W\rangle d \sigma \\
& =\langle F(Y, Z) Y, W\rangle-\int_{0}^{1}\langle F(Y, \sigma Z) Y, W\rangle d \sigma
\end{aligned}
$$

and then

$$
\begin{aligned}
& \frac{d}{d t} \int_{0}^{1}\langle F(Y, \sigma Z) Z, Y\rangle d \sigma \\
&=\langle F(Y, Z) Y, W\rangle+\int_{0}^{1}\langle J(F(Y, \sigma Z) Y \mid Y) Z, Z\rangle d \sigma \\
&=\langle F(Y, Z) Y, W\rangle+\int_{0}^{1}\langle F(Y, \sigma Z) Z, Z\rangle d \sigma \\
& \quad+\int_{0}^{1}\langle\{J(F(Y, \sigma Z) Y \mid Y)-F(Y, \sigma Z)\} Z, Z\rangle d \sigma \\
& \leqq\langle F(Y, Z) Y, W\rangle+\left\|\int_{0}^{1} F(Y, \sigma Z) d \sigma\right\|\langle Z, Z\rangle,
\end{aligned}
$$

since $J(F Y \mid Y)-F$ is negative-definite from assumption (vi).

\section{Proof of Theorem 2}

For the proof of the main stability theorem, it will be convenient to consider instead of (4) the equivalent system

$$
\begin{gathered}
\dot{X}=Y, \quad \dot{Y}=Z, \quad \dot{Z}=W, \\
\dot{W}=-F(Y, Z) W-\Phi(Z)-G(Y)-H(X) \\
+\int_{t-r}^{t} J_{G}(Y(s)) Z(s) d s+\int_{t-r}^{t} J_{H}(X(s)) Y(s) d s .
\end{gathered}
$$

The proof of Theorem 2 depends on a scalar differentiable function $V\left(X_{t}, Y_{t}, Z_{t}, W_{t}\right)$; now we define the Lyapunov functional $V$ as

$$
\begin{aligned}
2 V( & \left.X_{t}, Y_{t}, Z_{t}, W_{t}\right) \\
= & 2 d_{2} \int_{0}^{1}\langle H(\sigma X), X\rangle d \sigma+d_{2}\left\langle\alpha_{2} Y, Y\right\rangle \\
& -d_{1}\left\langle\alpha_{4} Y, Y\right\rangle+2 \int_{0}^{1}\langle G(\sigma Y), Y\rangle d \sigma \\
& +2 d_{1} \int_{0}^{1}\langle\Phi(\sigma Z), Z\rangle d \sigma \\
& -d_{2}\langle Z, Z\rangle+2 \int_{0}^{1}\langle\sigma F(Y, \sigma Z) Z, Z\rangle d \sigma+d_{1}\langle W, W\rangle \\
& +2\langle H(X), Y\rangle+2 d_{1}\langle H(X), Z\rangle \\
& +2 d_{2} \int_{0}^{1}\langle F(Y, \sigma Z) Z, Y\rangle d \sigma \\
& +2 d_{1}\langle G(Y), Z\rangle+2 d_{2}\langle Y, W\rangle+2\langle Z, W\rangle \\
& +2 \mu \int_{-r}^{0} \int_{t+s}^{t}\|Y(\theta)\|^{2} d \theta d s \\
& +2 \lambda \int_{-r}^{0} \int_{t+s}^{t}\|Z(\theta)\|^{2} d \theta d s,
\end{aligned}
$$

where $\mu$ and $\lambda$ are positive constants, which will be determined later. Let

$$
F_{1}(Y, Z)=\int_{0}^{1} F(Y, \sigma Z) d \sigma .
$$

Since $\lambda_{i}(F(Y, Z)) \geq \alpha_{1}>0$, for all $Y, Z \in R^{n}$, it follows that

$$
\lambda_{i}\left(F_{1}(Y, Z)\right) \geq \alpha_{1}>0, \quad \forall Y, Z \in R^{n} .
$$

Further we define

$$
\Gamma(Y)=\int_{0}^{1} J_{G}(\sigma Y) d \sigma,
$$

and then it follows from (ii) and (iv) that

$$
\lambda_{i}(\Gamma(Y)) \geq \frac{\alpha_{3} \alpha_{4}^{2}}{\alpha_{4}^{\prime 2}}>0,
$$

for all $Y \in R^{n}$, and

$$
0 \leq \lambda_{i}\left(J_{G}(Y)-\Gamma(Y)\right) \leq \delta_{1}, \quad \forall Y \in R^{n}
$$

Since

$$
\frac{\partial}{\partial \sigma} \Phi(\sigma Z)=J_{\Phi}(\sigma Z) Z, \quad \Phi(0)=0
$$

then

$$
\Phi(Z)=\int_{0}^{1} J_{\Phi}(\sigma Z) Z d \sigma
$$


Therefore

$$
\begin{aligned}
& 2 d_{1} \int_{0}^{1}\langle\Phi(\sigma Z), Z\rangle d \sigma \\
& \quad=2 d_{1} \int_{0}^{1} \int_{0}^{1}\left\langle J_{\Phi}\left(\sigma_{1} \sigma_{2} Z\right) \sigma_{2} Z, Z\right\rangle d \sigma_{1} d \sigma_{2} \\
& \quad=2 d_{1} \int_{0}^{1}\left[\int_{0}^{1}\left\langle J_{\Phi}\left(\sigma_{1} \widetilde{Z}\right) \widetilde{Z}, Z\right\rangle d \sigma_{1}\right] d \sigma_{2} \\
& \quad \geq 2 d_{1} \int_{0}^{1} \alpha_{2}\langle\widetilde{Z}, Z\rangle d \sigma_{2}, \quad \text { by (ix) } \\
& \quad=2 d_{1} \int_{0}^{1} \alpha_{2}\langle Z, Z\rangle \sigma_{2} d \sigma_{2}=d_{1} \alpha_{2}\langle Z, Z\rangle .
\end{aligned}
$$

Also since

$2 \mu \int_{-r}^{0} \int_{t+s}^{t}\|Y(\theta)\|^{2} d \theta d s, \quad 2 \lambda \int_{-r}^{0} \int_{t+s}^{t}\|Z(\theta)\|^{2} d \theta d s$

are nonnegative, consequently we obtain

$$
\begin{aligned}
2 V( & \left.X_{t}, Y_{t}, Z_{t}, W_{t}\right) \\
\geq & 2 d_{2} \int_{0}^{1}\langle H(\sigma X), X\rangle d \sigma+d_{2}\left\langle\alpha_{2} Y, Y\right\rangle-d_{1}\left\langle\alpha_{4} Y, Y\right\rangle \\
& +2 \int_{0}^{1}\langle G(\sigma Y), Y\rangle d \sigma+\left(\alpha_{2} d_{1}-d_{2}\right)\langle Z, Z\rangle \\
& +2 \int_{0}^{1}\langle\sigma F(Y, \sigma Z) Z, Z\rangle d \sigma+d_{1}\langle W, W\rangle \\
& +2\langle H(X), Y\rangle \\
& +2 d_{1}\langle H(X), Z\rangle+2 d_{2} \int_{0}^{1}\langle F(Y, \sigma Z) Z, Y\rangle d \sigma \\
& +2 d_{1}\langle G(Y), Z\rangle+2 d_{2}\langle Y, W\rangle+2\langle Z, W\rangle .
\end{aligned}
$$

Then we can find

$$
\begin{aligned}
2 V \geq & 2 d_{2} \int_{0}^{1}\langle H(\sigma X), X\rangle d \sigma-\left\|\Gamma^{-1 / 2} H(X)\right\|^{2}+d_{2}\left\langle\alpha_{2} Y, Y\right\rangle \\
& -d_{1}\left\langle\alpha_{4} Y, Y\right\rangle-d_{2}^{2}\left\|F_{1}^{1 / 2} Y\right\|^{2}+2 \int_{0}^{1}\langle G(\sigma Y), Y\rangle d \sigma \\
& -\left\|\Gamma^{1 / 2} Y\right\|^{2}+\left(\alpha_{2} d_{1}-d_{2}\right)\|Z\|^{2}-d_{1}^{2}\left\|\Gamma^{1 / 2} Z\right\|^{2} \\
& +2 \int_{0}^{1}\langle\sigma F(Y, \sigma Z) Z, Z\rangle d \sigma-\left\|F_{1}^{1 / 2} Z\right\|^{2}+d_{1}\|W\|^{2} \\
& -\left\|F_{1}^{-1 / 2} W\right\|^{2}+\left\|F_{1}^{-1 / 2} W+F_{1}^{1 / 2} Z+d_{2} F_{1}^{1 / 2} Y\right\|^{2} \\
& +\left\|\Gamma^{-1 / 2} H(X)+\Gamma^{1 / 2} Y+d_{1} \Gamma^{1 / 2} Z\right\|^{2} .
\end{aligned}
$$

The matrices $F_{1}$ and $\Gamma$ are symmetric because $F$ and $J_{G}$ are symmetric. The eigenvalues of $F_{1}$ and $\Gamma$ are positive because of (22) and (24).

Consequently the square roots $F_{1}^{1 / 2}$ and $\Gamma^{1 / 2}$ exist; these are again symmetric and nonsingular for all $Y, Z \in R^{n}$.

Therefore we get

$$
\begin{aligned}
2 V \geq & 2 d_{2} \int_{0}^{1}\langle H(\sigma X), X\rangle d \sigma-\left\langle\Gamma^{-1} H(X), H(X)\right\rangle \\
& +d_{2}\left\langle\alpha_{2} Y, Y\right\rangle-d_{1}\left\langle\alpha_{4} Y, Y\right\rangle-d_{2}^{2}\left\langle F_{1} Y, Y\right\rangle \\
& +2 \int_{0}^{1}\langle G(\sigma Y), Y\rangle d \sigma-\langle\Gamma Y, Y\rangle+\left(\alpha_{2} d_{1}-d_{2}\right)\|Z\|^{2} \\
& -d_{1}^{2}\langle\Gamma Z, Z\rangle+2 \int_{0}^{1}\langle\sigma F(Y, \sigma Z) Z, Z\rangle d \sigma-\left\langle F_{1} Z, Z\right\rangle \\
& +d_{1}\|W\|^{2}-\left\langle F_{1}^{-1} W, W\right\rangle .
\end{aligned}
$$

From $\lambda_{i}\left(F_{1}^{-1}\right) \leq 1 / \alpha_{1}$ and $\lambda_{i}\left(\Gamma^{-1}\right) \leq \alpha_{4}^{\prime 2} / \alpha_{3} \alpha_{4}^{2}$, because of (22) and (24), we get from Lemma 3 and Cauchy-Schwartz inequality that

$$
\begin{aligned}
2 V \geq & 2 d_{2} \int_{0}^{1}\langle H(\sigma X), X\rangle d \sigma-\left\langle\Gamma^{-1} H(X), H(X)\right\rangle \\
& +2 \int_{0}^{1}\langle G(\sigma Y), Y\rangle d \sigma-\langle\Gamma Y, Y\rangle \\
& +\left(\alpha_{2} d_{2}-\alpha_{4} d_{1}-d_{2}^{2}\left\|F_{1}\right\|\right)\|Y\|^{2} \\
& +\left(\alpha_{2} d_{1}-d_{2}-d_{1}^{2}\|\Gamma\|\right)\|Z\|^{2} \\
& +2 \int_{0}^{1}\langle\sigma F(Y, \sigma Z) Z, Z\rangle d \sigma-\left\langle F_{1} Z, Z\right\rangle \\
& +\left(d_{1}-\frac{1}{\alpha_{1}}\right)\|W\|^{2} .
\end{aligned}
$$

From the definitions of $d_{1}, d_{2}$ in (11), it follows that

$$
2 V\left(X_{t}, Y_{t}, Z_{t}, W_{t}\right) \geq V_{1}+V_{2}+V_{3}+\varepsilon\|W\|^{2},
$$

where

$$
\begin{aligned}
V_{1}:= & 2 d_{2} \int_{0}^{1}\langle H(\sigma X), X\rangle d \sigma-\left\langle\Gamma^{-1} H(X), H(X)\right\rangle, \\
V_{2}:= & \left(\alpha_{2} d_{2}-\alpha_{4} d_{1}-d_{2}^{2}\left\|F_{1}\right\|\right)\|Y\|^{2} \\
& +2 \int_{0}^{1}\langle G(\sigma Y), Y\rangle d \sigma-\langle\Gamma Y, Y\rangle, \\
V_{3}:= & \left(\alpha_{2} d_{1}-d_{2}-d_{1}^{2}\|\Gamma\|\right)\|Z\|^{2} \\
& +2 \int_{0}^{1}\langle\sigma F(Y, \sigma Z) Z, Z\rangle d \sigma-\left\langle F_{1} Z, Z\right\rangle .
\end{aligned}
$$




$$
\begin{aligned}
& \text { Since } \\
& \frac{\partial}{\partial \sigma_{1}}\left\langle H\left(\sigma_{1} X\right), H\left(\sigma_{1} X\right)\right\rangle=2\left\langle J_{H}\left(\sigma_{1} X\right) X, H\left(\sigma_{1} X\right)\right\rangle,
\end{aligned}
$$

by integrating both sides from $\sigma_{1}=0$ to $\sigma_{1}=1$ and because of $H(0)=0$, we obtain

$$
\langle H(X), H(X)\rangle=2 \int_{0}^{1}\left\langle J_{H}\left(\sigma_{1} X\right) X, H\left(\sigma_{1} X\right)\right\rangle d \sigma_{1} .
$$

Thus

$$
\begin{aligned}
V_{1}= & 2 d_{2} \int_{0}^{1}\langle H(\sigma X), X\rangle d \sigma \\
& -2 \Gamma^{-1} \int_{0}^{1}\left\langle J_{H}\left(\sigma_{1} X\right) X, H\left(\sigma_{1} X\right)\right\rangle d \sigma_{1} \\
= & 2 \int_{0}^{1}\left\langle H\left(\sigma_{1} X\right),\left\{d_{2} I-\Gamma^{-1} J_{H}\left(\sigma_{1} X\right)\right\} X\right\rangle d \sigma_{1} .
\end{aligned}
$$

But from

$$
\begin{aligned}
& \frac{\partial}{\partial \sigma_{2}}\left\langle H\left(\sigma_{1} \sigma_{2} X\right),\left\{d_{2} I-\Gamma^{-1} J_{H}\left(\sigma_{1} X\right)\right\} X\right\rangle \\
& \quad=\left\langle\sigma_{1} J_{H}\left(\sigma_{1} \sigma_{2} X\right) X,\left\{d_{2} I-\Gamma^{-1} J_{H}\left(\sigma_{1} X\right)\right\} X\right\rangle,
\end{aligned}
$$

by integrating both sides from $\sigma_{2}=0$ to $\sigma_{2}=1$ and because of $H(0)=0$, we find

$$
\begin{aligned}
& \left\langle H\left(\sigma_{1} \sigma_{2} X\right),\left\{d_{2} I-\Gamma^{-1} J_{H}\left(\sigma_{1} X\right)\right\} X\right\rangle \\
& \quad=\int_{0}^{1} \sigma_{1}\left\langle J_{H}\left(\sigma_{1} \sigma_{2} X\right) X,\left\{d_{2} I-\Gamma^{-1} J_{H}\left(\sigma_{1} X\right)\right\} X\right\rangle d \sigma_{2} .
\end{aligned}
$$
have

Therefore by using (11), (24), (vii), (viii), and Lemma 3, we

$$
\begin{gathered}
V_{1}=2 \int_{0}^{1} \int_{0}^{1} \sigma_{1}\left\langle J_{H}\left(\sigma_{1} \sigma_{2} X\right) X,\right. \\
\left.\left\{d_{2} I-\Gamma^{-1} J_{H}\left(\sigma_{1} X\right)\right\} X\right\rangle d \sigma_{2} d \sigma_{1} \\
=2 \int_{0}^{1} \int_{0}^{1} \sigma_{1}\left\langle J_{H}\left(\sigma_{1} \sigma_{2} X\right)\left\{d_{2} I-\Gamma^{-1} J_{H}\left(\sigma_{1} X\right)\right\} X,\right. \\
X\rangle d \sigma_{2} d \sigma_{1}
\end{gathered}
$$

$$
\begin{aligned}
& \geq 2 \varepsilon \int_{0}^{1} \int_{0}^{1}\left\langle J_{H}\left(\sigma_{1} \sigma_{2} X\right) \sigma_{1} X, X\right\rangle d \sigma_{2} d \sigma_{1} \\
& +\frac{2 \alpha_{4}^{\prime 2}}{\alpha_{3} \alpha_{4}^{2}} \int_{0}^{1} \int_{0}^{1} \sigma_{1}\left\langle J_{H}\left(\sigma_{1} \sigma_{2} X\right) X\right. \\
& \left.\qquad\left\{\alpha_{4} I-J_{H}\left(\sigma_{1} X\right)\right\} X\right\rangle d \sigma_{2} d \sigma_{1} \\
& \geq 2 \varepsilon \int_{0}^{1}\left[\int_{0}^{1}\left\langle J_{H}\left(\sigma_{2} \widetilde{X}\right) \widetilde{X}, X\right\rangle d \sigma_{2}\right] d \sigma_{1} \\
& \geq 2 \varepsilon \int_{0}^{1} \alpha_{4}^{\prime}\langle\widetilde{X}, X\rangle d \sigma_{1}=2 \varepsilon \int_{0}^{1} \alpha_{4}^{\prime}\langle X, X\rangle \sigma_{1} d \sigma_{1} \\
& =\varepsilon \alpha_{4}^{\prime}\langle X, X\rangle=\varepsilon \alpha_{4}^{\prime}\|X\|^{2} .
\end{aligned}
$$

To estimate $V_{2}$ we need

$$
\begin{aligned}
\alpha_{2} d_{2} & -\alpha_{4} d_{1}-d_{2}^{2}\left\|F_{1}\right\| \\
= & d_{2}\left\{\alpha_{2}-d_{1}\left\|J_{G}(Y)\right\|-d_{2}\left\|F_{1}\right\|\right\} \\
& +d_{1}\left\{d_{2}\left\|J_{G}(Y)\right\|-\alpha_{4}\right\} \\
\geq & d_{2}\left\{\alpha_{2}-d_{1}\left\|J_{G}(Y)\right\|-d_{2}\left\|F_{1}\right\|\right\},
\end{aligned}
$$

since from (11) and (ii) we find that

$$
d_{2}\left\|J_{G}(Y)\right\|-\alpha_{4}>\left(\varepsilon+\frac{\alpha_{4}^{\prime 2}}{\alpha_{3} \alpha_{4}}\right) \frac{\alpha_{3} \alpha_{4}^{2}}{\alpha_{4}^{\prime 2}}-\alpha_{4}=\varepsilon \frac{\alpha_{3} \alpha_{4}^{2}}{\alpha_{4}^{\prime 2}}>0 .
$$

Now

$$
\alpha_{2}-d_{1}\left\|J_{G}(Y)\right\|-d_{2}\left\|F_{1}\right\|
$$

$$
\begin{aligned}
= & \alpha_{2}-\frac{1}{\alpha_{1}}\left\|J_{G}(Y)\right\|-\frac{\alpha_{4}^{\prime 2}}{\alpha_{3} \alpha_{4}}\left\|F_{1}\right\| \\
& -\varepsilon\left\{\left\|J_{G}(Y)\right\|+\left\|F_{1}\right\|\right\} \\
= & \frac{1}{\alpha_{1} \alpha_{3} \alpha_{4}}\left[\alpha_{3} \alpha_{4}\left\{\alpha_{1} \alpha_{2}-\left\|J_{G}(Y)\right\|\right\}-\alpha_{1} \alpha_{4}^{\prime 2}\left\|F_{1}\right\|\right] \\
& -\varepsilon\left\{\left\|J_{G}(Y)\right\|+\left\|F_{1}\right\|\right\} \\
\geq & \frac{\Delta}{\alpha_{1} \alpha_{3} \alpha_{4}}-\varepsilon\left(\alpha_{1} \alpha_{2}+\alpha_{2} \alpha_{3} \alpha_{4} \alpha_{4}^{\prime-2}\right), \quad \text { from (iii). }
\end{aligned}
$$

Thus we obtain from (viii)

$$
\alpha_{2}-d_{1}\left\|J_{G}(Y)\right\|-d_{2}\left\|F_{1}\right\| \geq \frac{\Delta}{\alpha_{1} \alpha_{3} \alpha_{4}}-\varepsilon D_{0} .
$$

From the identity

$\int_{0}^{1} \sigma\left\langle J_{G}(\sigma Y) Y, Y\right\rangle d \sigma \equiv\langle G(Y), Y\rangle-\int_{0}^{1}\langle G(\sigma Y), Y\rangle d \sigma$, 
we get from (25) and by Lemma 3

$$
\begin{aligned}
2 \int_{0}^{1} & \langle G(\sigma Y), Y\rangle d \sigma-\langle G(Y), Y\rangle \\
& =\int_{0}^{1}\langle G(\sigma Y), Y\rangle d \sigma-\int_{0}^{1} \sigma\left\langle J_{G}(\sigma Y) Y, Y\right\rangle d \sigma \\
& =\int_{0}^{1} \sigma\langle\Gamma(\sigma Y) Y, Y\rangle d \sigma-\int_{0}^{1} \sigma\left\langle J_{G}(\sigma Y) Y, Y\right\rangle d \sigma \\
& =-\int_{0}^{1} \sigma\left\langle\left\{J_{G}(\sigma Y)-\Gamma(\sigma Y)\right\} Y, Y\right\rangle d \sigma \\
& \geq-\frac{1}{2} \delta_{1}\|Y\|^{2} .
\end{aligned}
$$

So we have from (9) and (11)

$$
\begin{aligned}
V_{2} & \geq d_{2}\left(\frac{\Delta}{\alpha_{1} \alpha_{3} \alpha_{4}}-\varepsilon D_{0}\right)\|Y\|^{2}-\frac{1}{2} \delta_{1}\|Y\|^{2} \\
& \geq\left\{\frac{\alpha_{4}^{\prime 2}}{\alpha_{3} \alpha_{4}}\left(\frac{\Delta}{\alpha_{1} \alpha_{3} \alpha_{4}}-\varepsilon D_{0}\right)-\frac{1}{2} \delta_{1}\right\}\|Y\|^{2} \\
& \geq \frac{1}{4}\left(\frac{2 \alpha_{4}^{\prime 2} \Delta}{\alpha_{1} \alpha_{3}^{2} \alpha_{4}^{2}}-\delta_{1}\right)\|Y\|^{2},
\end{aligned}
$$

since $\varepsilon<\left(\alpha_{3} \alpha_{4} / 4 \alpha_{4}^{\prime 2} D_{0}\right)\left(2 \alpha_{4}^{\prime 2} \Delta / \alpha_{1} \alpha_{3}^{2} \alpha_{4}^{2}-\delta_{1}\right)$.

To estimate $V_{3}$ we need

$$
\begin{aligned}
\alpha_{2} d_{1} & -d_{2}-d_{1}^{2}\|\Gamma\| \\
= & d_{1}\left\{\alpha_{2}-d_{1}\|\Gamma\|-d_{2}\left\|F_{1}\right\|\right\} \\
& +d_{2}\left\{d_{1}\left\|F_{1}\right\|-1\right\} \\
\geq & d_{1}\left\{\alpha_{2}-d_{1}\|\Gamma\|-d_{2}\left\|F_{1}\right\|\right\} \\
\geq & d_{1}\left\{\alpha_{2}-d_{1}\left\|J_{G}(Y)\right\|-d_{2}\left\|F_{1}\right\|\right\} \\
\geq & \frac{1}{\alpha_{1}}\left(\frac{\Delta}{\alpha_{1} \alpha_{3} \alpha_{4}}-\varepsilon D_{0}\right),
\end{aligned}
$$

by (11), (25), and (45). So from the identity

$$
\begin{aligned}
\int_{0}^{1} \sigma\langle F(Y, \sigma Z) Z, Z\rangle d \sigma \equiv & \int_{0}^{1}\langle F(Y, \sigma Z) Z, Z\rangle d \sigma \\
& -\int_{0}^{1} \sigma\left\langle F_{1}(Y, \sigma Z) Z, Z\right\rangle d \sigma
\end{aligned}
$$

we find

$$
\begin{aligned}
& 2 \int_{0}^{1} \sigma\langle F(Y, \sigma Z) Z, Z\rangle d \sigma-\left\langle F_{1} Z, Z\right\rangle \\
& \quad=\int_{0}^{1} \sigma\langle F(Y, \sigma Z) Z, Z\rangle d \sigma-\int_{0}^{1} \sigma\left\langle F_{1}(Y, \sigma Z) Z, Z\right\rangle d \sigma \\
& \quad=-\int_{0}^{1} \sigma\left\langle\left\{F(Y, \sigma Z)-F_{1}(Y, \sigma Z)\right\} Z, Z\right\rangle d \sigma \\
& \quad \geq-\frac{1}{2} \delta_{2}\|Z\|^{2}, \quad \text { by (v). }
\end{aligned}
$$

Thus from (9), we obtain

$$
\begin{aligned}
V_{3} & \geq\left\{\frac{1}{\alpha_{1}}\left(\frac{\Delta}{\alpha_{1} \alpha_{3} \alpha_{4}}-\varepsilon D_{0}\right)-\frac{1}{2} \delta_{2}\right\}\|Z\|^{2} \\
& \geq \frac{1}{4}\left(\frac{2 \Delta}{\alpha_{1}^{2} \alpha_{3} \alpha_{4}}-\delta_{2}\right)\|Z\|^{2},
\end{aligned}
$$

since $\varepsilon<\left(\alpha_{1} / 4 D_{0}\right)\left(2 \Delta / \alpha_{1}^{2} \alpha_{3} \alpha_{4}-\delta_{2}\right)$. Then it follows that

$$
\begin{aligned}
& 2 V\left(X_{t}, Y_{t}, Z_{t}, W_{t}\right) \\
& \geq \varepsilon \alpha_{4}^{\prime}\|X\|^{2}+\frac{1}{4}\left(\frac{2 \alpha_{4}^{\prime 2} \Delta}{\alpha_{1} \alpha_{3}^{2} \alpha_{4}^{2}}-\delta_{1}\right)\|Y\|^{2} \\
& \quad+\frac{1}{4}\left(\frac{2 \Delta}{\alpha_{1}^{2} \alpha_{3} \alpha_{4}}-\delta_{2}\right)\|Z\|^{2}+\varepsilon\|W\|^{2} .
\end{aligned}
$$

Since the coefficients are positive constants from the definitions of $\delta_{1}, \delta_{2}$, and $\varepsilon$ in (iv), (v), and (9), then there exists a positive constant $D_{1}$ such that

$$
V\left(X_{t}, Y_{t}, Z_{t}, W_{t}\right) \geq D_{1}\left(\|X\|^{2}+\|Y\|^{2}+\|Z\|^{2}+\|W\|^{2}\right) .
$$

To prove that

$$
V\left(X_{t}, Y_{t}, Z_{t}, W_{t}\right) \leq D_{2}\left(\|X\|^{2}+\|Y\|^{2}+\|Z\|^{2}+\|W\|^{2}\right),
$$

by using the hypotheses of Theorem 2 we find

$$
\left\|F_{1}\right\| \leq \sqrt{n} \alpha_{2} \alpha_{3} \alpha_{4} \alpha_{4}^{\prime-2}, \quad \text { by (iii). }
$$

Since

$$
\frac{\partial \Phi(\sigma Z)}{\partial \sigma}=J_{\Phi}(\sigma Z) Z, \quad \Phi(0)=0,
$$

then from (ix) we have

$$
\begin{aligned}
\|\Phi(Z)\| & =\left\|\int_{0}^{1} J_{\Phi}(\sigma Z) Z d \sigma\right\| \leq \int_{0}^{1}\left\|J_{\Phi}(\sigma Z)\right\|\|Z\| d \sigma \\
& \leq \sqrt{n}\left(\alpha_{2}+\frac{\varepsilon \alpha_{3}^{3} \alpha_{4}^{2}}{\alpha_{4}^{\prime 4}}\right)\|Z\|,
\end{aligned}
$$


and also since

$$
\frac{\partial G(\sigma Y)}{\partial \sigma}=J_{G}(\sigma Y) Y, \quad G(0)=0,
$$

then from (iv) we have

$$
\begin{aligned}
\|G(Y)\| & =\left\|\int_{0}^{1} J_{G}(\sigma Y) Y d \sigma\right\| \leq \int_{0}^{1}\left\|J_{G}(\sigma Y)\right\|\|Y\| d \sigma \\
& \leq \alpha_{1} \alpha_{2} \sqrt{n}\|Y\| .
\end{aligned}
$$

Since

$$
\frac{\partial H(\sigma X)}{\partial \sigma}=J_{H}(\sigma X) X, \quad H(0)=0,
$$

then from (viii) we get

$$
\begin{aligned}
\|H(X)\| & =\left\|\int_{0}^{1} J_{H}(\sigma X) X d \sigma\right\| \leq \int_{0}^{1}\left\|J_{H}(\sigma X)\right\|\|X\| d \sigma \\
& \leq \alpha_{4} \sqrt{n}\|X\| .
\end{aligned}
$$

By using Cauchy-Schwartz inequality $|\langle u, v\rangle| \leq$ $(1 / 2)\left(\|u\|^{2}+\|v\|^{2}\right)$ and from

$$
\begin{aligned}
& 2 \mu \int_{-r}^{0} \int_{t+s}^{t}\|Y(\theta)\|^{2} d \theta d s \\
& \quad=2 \mu \int_{t-r}^{t}(\theta-t+r)\|Y(\theta)\|^{2} d \theta \\
& \leq 2 \mu\|Y\|^{2} \int_{t-r}^{t}(\theta-t+r) d \theta \\
& \quad=\mu r^{2}\|Y\|^{2}, \\
& 2 \lambda \int_{-r}^{0} \int_{t+s}^{t}\|Z(\theta)\|^{2} d \theta d s \\
& \quad=2 \lambda \int_{t-r}^{t}(\theta-t+r)\|Z(\theta)\|^{2} d \theta \\
& \quad \leq 2 \lambda\|Z\|^{2} \int_{t-r}^{t}(\theta-t+r) d \theta \\
& \quad=\lambda r^{2}\|Z\|^{2} .
\end{aligned}
$$

Hence there exists a positive constant $D_{2}$ satisfying

$$
V\left(X_{t}, Y_{t}, Z_{t}, W_{t}\right) \leq D_{2}\left(\|X\|^{2}+\|Y\|^{2}+\|Z\|^{2}+\|W\|^{2}\right) \text {. }
$$

Now from (19), (20), and Lemma 4, we have

$$
\begin{aligned}
\frac{d V}{d t}= & d_{2}\langle H(X), Y\rangle+d_{2}\left\langle\alpha_{2} Y, Z\right\rangle-d_{1}\left\langle\alpha_{4} Y, Z\right\rangle \\
& +\langle G(Y), Z\rangle+d_{1}\langle\Phi(Z), W\rangle \\
& -d_{2}\langle Z, W\rangle+\langle F(Y, Z) Z, W\rangle \\
& +d_{1}\langle W,-F(Y, Z) W-\Phi(Z)-G(Y)-H(X) \\
& \quad+\int_{t-r}^{t} J_{G}(Y(s)) Z(s) d s \\
& \left.\quad+\int_{t-r}^{t} J_{H}(X(s)) Y(s) d s\right\rangle \\
& +\left\langle J_{H}(X) Y, Y\right\rangle+\langle H(X), Z\rangle+d_{1}\left\langle J_{H}(X) Y, Z\right\rangle \\
& +d_{1}\langle H(X), W\rangle+d_{2}\langle F(Y, Z) Y, W\rangle \\
& +d_{2}\left\|F_{1}\right\|\langle Z, Z\rangle \\
& +d_{1}\langle W, G(Y)\rangle+d_{1}\left\langle J_{G}(Y) Z, Z\right\rangle \\
& +d_{2}\langle Y,-F(Y, Z) W \\
& \quad-\Phi(Z)-G(Y)-H(X)
\end{aligned}
$$$$
+\int_{t-r}^{t} J_{G}(Y(s)) Z(s) d s
$$$$
\left.+\int_{t-r}^{t} J_{H}(X(s)) Y(s) d s\right\rangle+\langle W, W\rangle
$$

$+\langle Z,-F(Y, Z) W$

$$
\begin{aligned}
& -\Phi(Z)-G(Y)-H(X)+\int_{t-r}^{t} J_{G}(Y(s)) Z(s) d s \\
& \left.+\int_{t-r}^{t} J_{H}(X(s)) Y(s) d s\right\rangle+d_{2}\langle Z, W\rangle+\mu r\|Y\|^{2}
\end{aligned}
$$

$-\mu \int_{t-r}^{t}\|Y(\theta)\|^{2} d \theta+\lambda r\|Z\|^{2}-\lambda \int_{t-r}^{t}\|Z(\theta)\|^{2} d \theta$.

Then we get

$$
\begin{aligned}
\frac{d V}{d t}= & d_{2}\left\langle\alpha_{2} Y, Z\right\rangle-d_{1}\left\langle\alpha_{4} Y, Z\right\rangle-d_{1}\langle W, F(Y, Z) W\rangle \\
& -d_{2}\langle Y, \Phi(Z)\rangle+\left\langle J_{H}(X) Y, Y\right\rangle \\
& +d_{2}\left\|F_{1}\right\|\langle Z, Z\rangle+d_{1}\left\langle J_{G}(Y) Z, Z\right\rangle+\langle W, W\rangle \\
& +d_{1}\left\langle J_{H}(X) Y, Z\right\rangle-\langle Z, \Phi(Z)\rangle-d_{2}\langle Y, G(Y)\rangle \\
& +\left\langle d_{1} W+Z+d_{2} Y, \int_{t-r}^{t} J_{H}(X(s)) Y(s) d s\right\rangle
\end{aligned}
$$




$$
\begin{aligned}
& +\left\langle d_{1} W+Z+d_{2} Y, \int_{t-r}^{t} J_{G}(Y(s)) Z(s) d s\right\rangle \\
& +\mu r\|Y\|^{2}-\mu \int_{t-r}^{t}\|Y(\theta)\|^{2} d \theta+\lambda r\|Z\|^{2} \\
& -\lambda \int_{t-r}^{t}\|Z(\theta)\|^{2} d \theta
\end{aligned}
$$

and it follows that

$$
\begin{aligned}
\frac{d V}{d t}= & \left\langle\alpha_{4} Y, Y\right\rangle-d_{2}\langle Y, G(Y)\rangle \\
& -\left\langle\alpha_{2} Z, Z\right\rangle+d_{1}\left\langle J_{G}(Y) Z, Z\right\rangle \\
& +d_{2}\left\|F_{1}\right\|\langle Z, Z\rangle-d_{1}\langle W, F(Y, Z) W\rangle+\langle W, W\rangle \\
& +\left\langle d_{1} W+Z+d_{2} Y, \int_{t-r}^{t} J_{H}(X(s)) Y(s) d s\right\rangle \\
& +\left\langle d_{1} W+Z+d_{2} Y, \int_{t-r}^{t} J_{G}(Y(s)) Z(s) d s\right\rangle \\
& +\mu r\|Y\|^{2}-\mu \int_{t-r}^{t}\|Y(\theta)\|^{2} d \theta+\lambda r\|Z\|^{2} \\
& -\lambda \int_{t-r}^{t}\|Z(\theta)\|^{2} d \theta+V_{4}+V_{5},
\end{aligned}
$$

where

$$
\begin{aligned}
V_{4}:= & d_{2}\left\langle\alpha_{2} Z, Y\right\rangle-d_{2}\langle Y, \Phi(Z)\rangle \\
& -\langle Z, \Phi(Z)\rangle+\left\langle\alpha_{2} Z, Z\right\rangle, \\
V_{5}:= & -d_{1}\left\langle\alpha_{4} Z, Y\right\rangle+d_{1}\left\langle J_{H}(X) Y, Z\right\rangle \\
& +\left\langle J_{H}(X) Y, Y\right\rangle-\left\langle\alpha_{4} Y, Y\right\rangle .
\end{aligned}
$$

But

$$
\begin{aligned}
V_{4}=-\int_{0}^{1}\left[\left\langle J_{\Phi}(\sigma Z) Z, Z\right\rangle-\left\langle\alpha_{2} Z, Z\right\rangle\right. \\
\left.+d_{2}\left\{\left\langle J_{\Phi}(\sigma Z) Z, Y\right\rangle-\left\langle\alpha_{2} Z, Y\right\rangle\right\}\right] d \sigma \\
=-\int_{0}^{1}\left\langle\left\{J_{\Phi}(\sigma Z)-\alpha_{2} I\right\} Z, Z\right\rangle d \sigma \\
-d_{2} \int_{0}^{1}\left\langle\left\{J_{\Phi}(\sigma Z)-\alpha_{2} I\right\} Z, Y\right\rangle d \sigma .
\end{aligned}
$$

Since $\lambda_{i}\left(\int_{0}^{1} J_{\Phi}(\sigma Z) d \sigma-\alpha_{2} I\right)$ is nonnegative by (ix), then from (11) we get

$$
\begin{aligned}
V_{4} & \leq \frac{d_{2}^{2}}{4} \int_{0}^{1}\left\langle\left\{J_{\Phi}(\sigma Z)-\alpha_{2} I\right\} Y, Y\right\rangle d \sigma \\
& \leq \frac{1}{4}\left(\varepsilon+\frac{\alpha_{4}^{\prime 2}}{\alpha_{3} \alpha_{4}}\right)^{2} \varepsilon_{0} \alpha_{3}^{3} \alpha_{4}^{2} \alpha_{4}^{\prime-4}\langle Y, Y\rangle \\
& =\frac{1}{4}\left(\varepsilon \alpha_{3} \alpha_{4} \alpha_{4}^{\prime-2}+1\right)^{2} \varepsilon_{0} \alpha_{3}\|Y\|^{2} \\
& \leq \varepsilon_{0} \alpha_{3}\|Y\|^{2},
\end{aligned}
$$

since $\varepsilon<\alpha_{4}^{\prime 2} / \alpha_{3} \alpha_{4}$ by (9). Also

$$
\begin{aligned}
V_{5}=- & \left\{\left\langle\alpha_{4} Y, Y\right\rangle-\left\langle J_{H}(X) Y, Y\right\rangle+d_{1}\left\langle\alpha_{4} Z, Y\right\rangle\right. \\
& \left.-d_{1}\left\langle J_{H}(X) Y, Z\right\rangle\right\} \\
=-\langle & \left.\left\{\alpha_{4} I-J_{H}(X)\right\} Y, Y\right\rangle-d_{1}\left\langle\left\{\alpha_{4} I-J_{H}(X)\right\} Y, Z\right\rangle .
\end{aligned}
$$
we get

But $\lambda_{i}\left(\alpha_{4} I-J_{H}(X)\right)$ is nonnegative by (viii) and from (11),

$$
\begin{aligned}
V_{4} & \leq \frac{d_{1}^{2}}{4}\left\langle\left\{\alpha_{4} I-J_{H}(X)\right\} Z, Z\right\rangle \\
& \leq \frac{1}{4}\left(\varepsilon+\frac{1}{\alpha_{1}}\right)^{2} \varepsilon D_{0} \alpha_{1}^{2}\langle Z, Z\rangle \\
& =\frac{1}{4}\left(\varepsilon \alpha_{1}+1\right)^{2} \varepsilon D_{0}\|Z\|^{2} \\
& \leq \varepsilon D_{0}\|Z\|^{2}, \quad \text { since } \varepsilon<\frac{1}{\alpha_{1}} .
\end{aligned}
$$

Therefore

$$
\begin{aligned}
\dot{V} \leq & -\left\{d_{2}\langle Y, G(Y)\rangle-\left\langle\alpha_{4} Y, Y\right\rangle\right\}+\varepsilon_{0} \alpha_{3}\|Y\|^{2} \\
& -\left(\left\langle\alpha_{2} Z, Z\right\rangle-d_{2}\left\|F_{1}\right\|\|Z\|^{2}-d_{1}\left\|J_{G}\right\|\|Z\|^{2}\right) \\
& -\left\{d_{1}\langle W, F(Y, Z) W\rangle-\langle W, W\rangle\right\}+\varepsilon D_{0}\|Z\|^{2} \\
& +\left\langle d_{1} W+Z+d_{2} Y, \int_{t-r}^{t} J_{H}(X(s)) Y(s) d s\right\rangle \\
& +\left\langle d_{1} W+Z+d_{2} Y, \int_{t-r}^{t} J_{G}(Y(s)) Z(s) d s\right\rangle \\
& +\mu r\|Y\|^{2}-\mu \int_{t-r}^{t}\|Y(\theta)\|^{2} d \theta \\
& +\lambda r\|Z\|^{2}-\lambda \int_{t-r}^{t}\|Z(\theta)\|^{2} d \theta .
\end{aligned}
$$


We know that $\langle Y, G(Y)\rangle=\langle Y, \Gamma(Y) Y\rangle$ and by Lemma 3, we get

$$
\begin{aligned}
\frac{d V}{d t} \leq & -\left(d_{2} \frac{\alpha_{3} \alpha_{4}^{2}}{\alpha_{4}^{\prime 2}}-\alpha_{4}\right)\|Y\|^{2}+\varepsilon_{0} \alpha_{3}\|Y\|^{2} \\
& -\left(\alpha_{2}-d_{1}\left\|J_{G}\right\|-d_{2}\left\|F_{1}\right\|\right)\|Z\|^{2}+\varepsilon D_{0}\|Z\|^{2} \\
& -\left\{\alpha_{1} d_{1}-1\right\}\|W\|^{2} \\
& +\left\langle d_{1} W+Z+d_{2} Y, \int_{t-r}^{t} J_{H}(X(s)) Y(s) d s\right\rangle \\
& +\left\langle d_{1} W+Z+d_{2} Y, \int_{t-r}^{t} J_{G}(Y(s)) Z(s) d s\right\rangle \\
& +\mu r\|Y\|^{2}-\mu \int_{t-r}^{t}\|Y(\theta)\|^{2} d \theta+\lambda r\|Z\|^{2} \\
& -\lambda \int_{t-r}^{t}\|Z(\theta)\|^{2} d \theta .
\end{aligned}
$$

Since $\left\|J_{H}(X)\right\| \leq \alpha_{4} \sqrt{n}$ by (viii) and by using CauchySchwartz inequality, we obtain

$$
\begin{aligned}
\left|\left\langle d_{1} W+Z+d_{2} Y, \int_{t-r}^{t} J_{H}(X(s)) Y(s) d s\right\rangle\right| \\
\leq\left\|d_{1} W+Z+d_{2} Y\right\|\left\|\int_{t-r}^{t} J_{H}(X(s)) Y(s) d s\right\| \\
\leq\left(d_{1}\|W\|+\|Z\|+d_{2}\|Y\|\right) \int_{t-r}^{t} \alpha_{4} \sqrt{n}\|Y(s)\| d s \\
\leq \frac{d_{1} \alpha_{4} \sqrt{n}}{2}\left(\|W\|^{2} r+\int_{t-r}^{t}\|Y(s)\|^{2} d s\right) \\
+\frac{\alpha_{4} \sqrt{n}}{2}\left(\|Z\|^{2} r+\int_{t-r}^{t}\|Y(s)\|^{2} d s\right) \\
+\frac{d_{2} \alpha_{4} \sqrt{n}}{2}\left(\|Y\|^{2} r+\int_{t-r}^{t}\|Y(s)\|^{2} d s\right) .
\end{aligned}
$$

Also, since $\left\|J_{G}(Y)\right\| \leq \alpha_{1} \alpha_{2} \sqrt{n}$ by (iii) and by using Cauchy-Schwartz inequality, we find

$$
\begin{aligned}
& \left|\left\langle d_{1} W+Z+d_{2} Y, \int_{t-r}^{t} J_{G}(Y(s)) Z(s) d s\right\rangle\right| \\
& \leq\left\|d_{1} W+Z+d_{2} Y\right\|\left\|\int_{t-r}^{t} J_{G}(Y(s)) Z(s) d s\right\| \\
& \leq\left(d_{1}\|W\|+\|Z\|+d_{2}\|Y\|\right) \int_{t-r}^{t} \alpha_{1} \alpha_{2} \sqrt{n}\|Z(s)\| d s \\
& \leq \frac{d_{1} \alpha_{1} \alpha_{2} \sqrt{n}}{2}\left(\|W\|^{2} r+\int_{t-r}^{t}\|Z(s)\|^{2} d s\right) \\
& +\frac{\alpha_{1} \alpha_{2} \sqrt{n}}{2}\left(\|Z\|^{2} r+\int_{t-r}^{t}\|Z(s)\|^{2} d s\right) \\
& +\frac{d_{2} \alpha_{1} \alpha_{2} \sqrt{n}}{2}\left(\|Y\|^{2} r+\int_{t-r}^{t}\|Z(s)\|^{2} d s\right) .
\end{aligned}
$$

Therefore it follows from (11) and (45) that

$$
\begin{aligned}
& \frac{d V}{d t} \leq-\left\{\left(\frac{\alpha_{4}^{2}}{\alpha_{4}^{\prime 2}} \varepsilon-\varepsilon_{0}\right) \alpha_{3}-\frac{d_{2} \alpha_{4} \sqrt{n}}{2} r\right. \\
& \left.-\frac{d_{2} \alpha_{1} \alpha_{2} \sqrt{n}}{2} r-\mu r\right\}\|Y\|^{2} \\
& -\left(\frac{\Delta}{2 \alpha_{1} \alpha_{3} \alpha_{4}}-\frac{\alpha_{4} \sqrt{n}}{2} r-\frac{\alpha_{1} \alpha_{2} \sqrt{n}}{2} r-\lambda r\right)\|Z\|^{2} \\
& -\left(\varepsilon-\frac{d_{1} \alpha_{4} \sqrt{n}}{2} r-\frac{d_{1} \alpha_{1} \alpha_{2} \sqrt{n}}{2} r\right)\|W\|^{2} \\
& +\left(\frac{d_{1} \alpha_{4} \sqrt{n}}{2}+\frac{d_{2} \alpha_{4} \sqrt{n}}{2}+\frac{\alpha_{4} \sqrt{n}}{2}-\mu\right) \int_{t-r}^{t}\|Y(s)\|^{2} d s \\
& +\left(\frac{d_{1} \alpha_{1} \alpha_{2} \sqrt{n}}{2}+\frac{d_{2} \alpha_{1} \alpha_{2} \sqrt{n}}{2}\right. \\
& \left.+\frac{\alpha_{1} \alpha_{2} \sqrt{n}}{2}-\lambda\right) \int_{t-r}^{t}\|Z(s)\|^{2} d s,
\end{aligned}
$$

and if we take

$$
\mu=\frac{\alpha_{4} \sqrt{n}}{2}\left(d_{1}+d_{2}+1\right), \quad \lambda=\frac{\alpha_{1} \alpha_{2} \sqrt{n}}{2}\left(d_{1}+d_{2}+1\right),
$$

then we have

$$
\begin{aligned}
& \frac{d V}{d t} \leq-\left\{\left(\frac{\alpha_{4}^{2}}{\alpha_{4}^{\prime 2}} \varepsilon-\varepsilon_{0}\right) \alpha_{3}-\frac{d_{2} \alpha_{4} \sqrt{n}}{2} r-\frac{d_{2} \alpha_{1} \alpha_{2} \sqrt{n}}{2} r\right. \\
& \left.-\frac{\alpha_{4} \sqrt{n}}{2}\left(d_{1}+d_{2}+1\right) r\right\}\|Y\|^{2} \\
& -\left\{\frac{\Delta}{2 \alpha_{1} \alpha_{3} \alpha_{4}}-\frac{\alpha_{4} \sqrt{n}}{2} r-\frac{\alpha_{1} \alpha_{2} \sqrt{n}}{2} r\right. \\
& \left.-\frac{\alpha_{1} \alpha_{2} \sqrt{n}}{2}\left(d_{1}+d_{2}+1\right) r\right\}\|Z\|^{2} \\
& -\left(\varepsilon-\frac{d_{1} \alpha_{4} \sqrt{n}}{2} r-\frac{d_{1} \alpha_{1} \alpha_{2} \sqrt{n}}{2} r\right)\|W\|^{2} .
\end{aligned}
$$

Therefore if

$$
\begin{aligned}
r<\min \left[\frac{\varepsilon}{d_{1} \sqrt{n}\left(\alpha_{4}+\alpha_{1} \alpha_{2}\right)},\right. \\
\\
\\
\frac{\frac{\Delta}{2 \alpha_{1} \alpha_{3} \alpha_{4} \sqrt{n}\left\{\alpha_{4}+\alpha_{1} \alpha_{2}\left(d_{1}+d_{2}+2\right)\right\}},}{} \\
\left.\frac{\left(\left(\alpha_{4}^{2} / \alpha_{4}^{\prime 2}\right) \varepsilon-\varepsilon_{0}\right) \alpha_{3}}{\alpha_{4} \sqrt{n}\left(d_{1}+2 d_{2}+1\right)+\alpha_{1} \alpha_{2} d_{2} \sqrt{n}}\right],
\end{aligned}
$$


we obtain

$$
\frac{d V}{d t}\left(X_{t}, Y_{t}, Z_{t}, W_{t}\right) \leq-\alpha\left(\|Y\|^{2}+\|Z\|^{2}+\|W\|^{2}\right),
$$

for some $\alpha>0$. Therefore from (54), (64), and (81) the functional $V\left(X_{t}, Y_{t}, Z_{t}, W_{t}\right)$ satisfies all the conditions of Theorem 1, so that the zero solution of (4) is uniformly stable.

Thus the proof of Theorem 2 is now complete.

\section{Conflict of Interests}

The authors declare that there is no conflict of interests regarding the publication of this paper.

\section{References}

[1] A. M. Lyapunov, Stability of Motion, Academic Press, New York, NY, USA, 1966.

[2] N. N. Krasovskii, "On conditions of inversion of A. M. Lyapunov's theorems on instability for stationary systems of differential equations," Doklady Akademii Nauk SSSR (N.S.), vol. 101, pp. 17-20, 1955 (Russian).

[3] T. Yoshizawa, Stability Theory by Lyapunov's Second Method, The Mathematical Society of Japan, 1966.

[4] R. Reissig, G. Sansone, and R. Conti, Nonlinear Differential Equations of Higher-Order, Noordhoff International Publishing, Leyden, The Netherlands, 1974, translated from the German.

[5] A. M. Abou-El-Ela and A. I. Sadek, "A stability result for the solutions of a certain system of fourth-order differential equations," Annals of Differential Equations, vol. 6, no. 2, pp. 131$141,1990$.

[6] A. M. A. Abou-El-Ela and A. I. Sadek, "A stability theorem for a certain fourth-order vector differential equation," Annals of Differential Equations, vol. 10, no. 2, pp. 125-134, 1994.

[7] A. M. A. Abou-El-Ela and A. I. Sadek, "On the asymptotic behaviour of solutions of certain non-autonomous differential equations," Journal of Mathematical Analysis and Applications, vol. 237, no. 1, pp. 360-375, 1999.

[8] H. Bereketoglu and C. Kart, "Some results for a certain third-order nonlinear ordinary differential equation," Bulletin Mathématique de la Société des Sciences Mathématiques de Roumanie, vol. 39, no. 1-4, pp. 77-83, 1996.

[9] A. I. Sadek, "On the stability of solutions of certain fourthorder delay differential equations," Applied Mathematics and Computation, vol. 148, no. 2, pp. 587-597, 2004.

[10] C. Tunç, "Some stability results for the solutions of certain fourthorder delay differential equations," Journal of Difference Equations and Applications, vol. 4, pp. 165-174, 2005.

[11] C. Tunç, "On stability of solutions of certain fourth-order delay differential equations," Applied Mathematics and Mechanics. English Edition, vol. 27, no. 8, pp. 1141-1148, 2006.

[12] C. Tunç, "On asymptotic stability of solutions to third-order nonlinear differential equations with retarded argument," Communications in Applied Analysis, vol. 11, no. 3-4, pp. 515-527, 2007.

[13] C. Tunç, "On the stability of solutions to a certain fourth-order delay differential equation," Nonlinear Dynamics, vol. 51, no. 1-2, pp. 71-81, 2008.

[14] A. M. A. Abou-El-Ela, A. I. Sadek, and A. M. Mahmoud, "On the stability of solutions to a certain fourth-order vector delay differential equation," Annals of Differential Equations, vol. 28, no. 1, pp. 1-10, 2012.

[15] T. A. Burton, Stability and Periodic Solutions of Ordinary and Functional Differential Equations, Academic Press, New York, NY, USA, 1985.

[16] R. Bellman, Introduction to Matrix Analysis, Classics in Applied Mathematics 19, reprint of the second edition (1970), with a foreword by Gene Golub, Society for Industrial and Applied Mathematics (SIAM), Philadelphia, Pa, USA, 1997. 


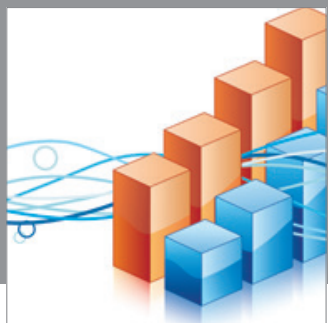

Advances in

Operations Research

mansans

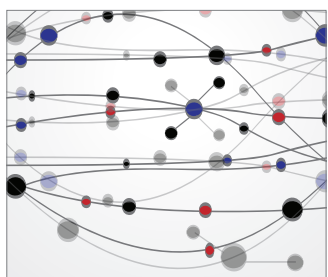

The Scientific World Journal
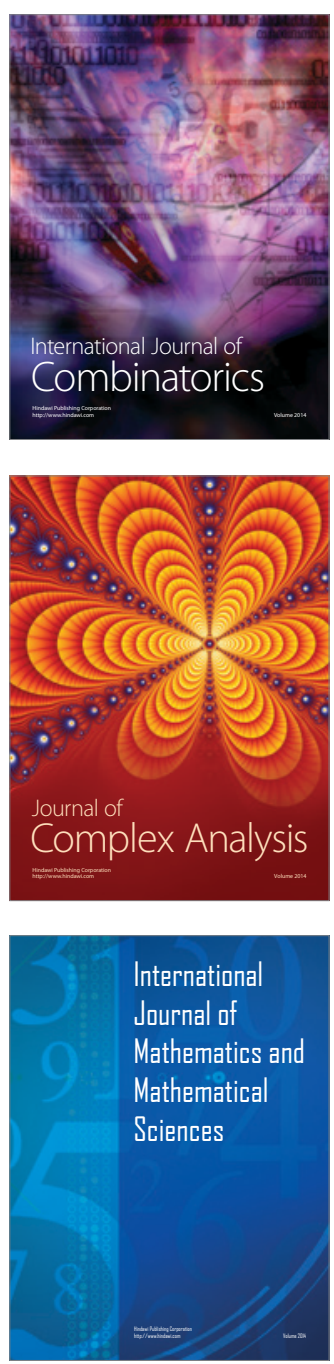
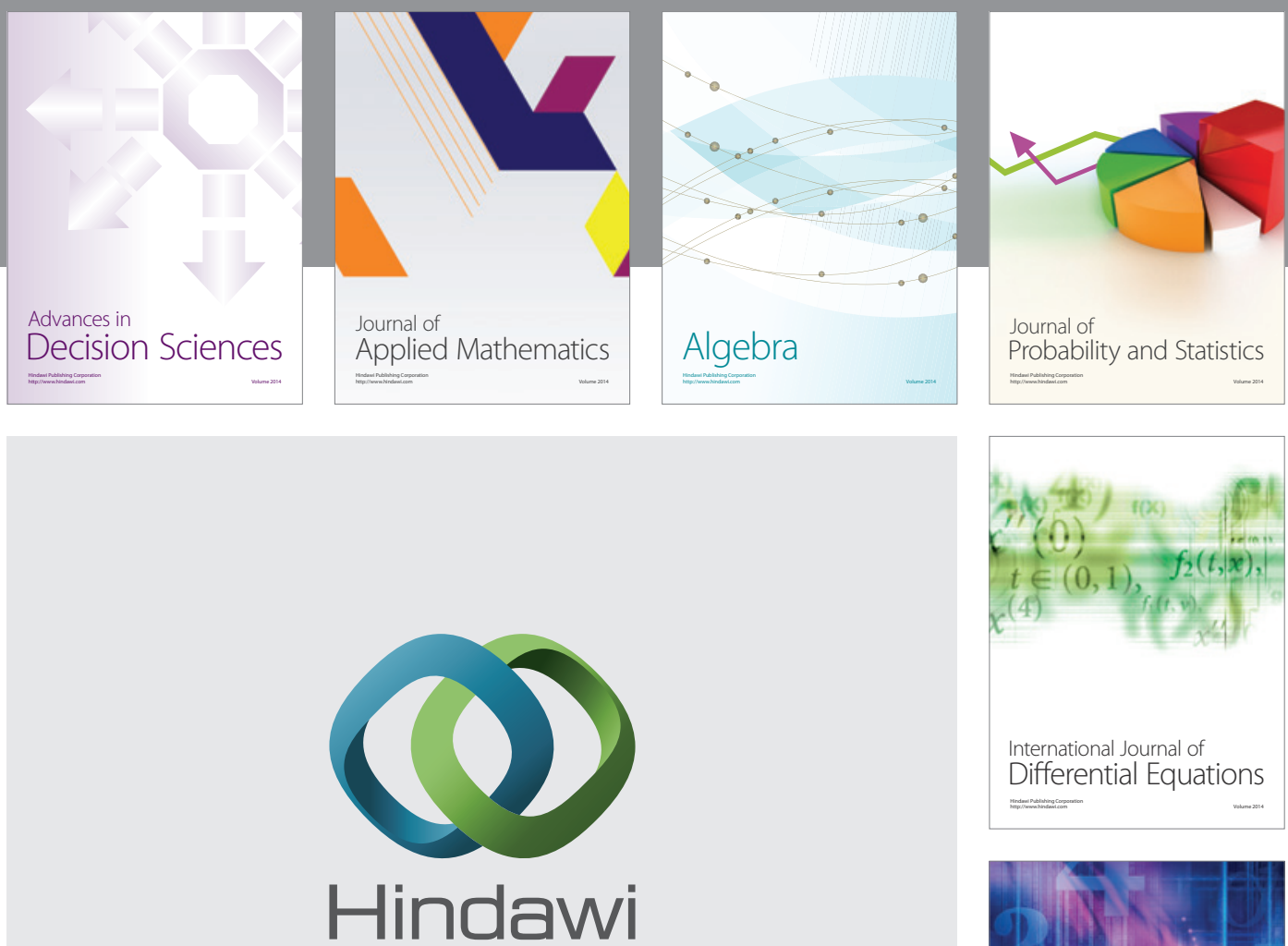

Submit your manuscripts at http://www.hindawi.com
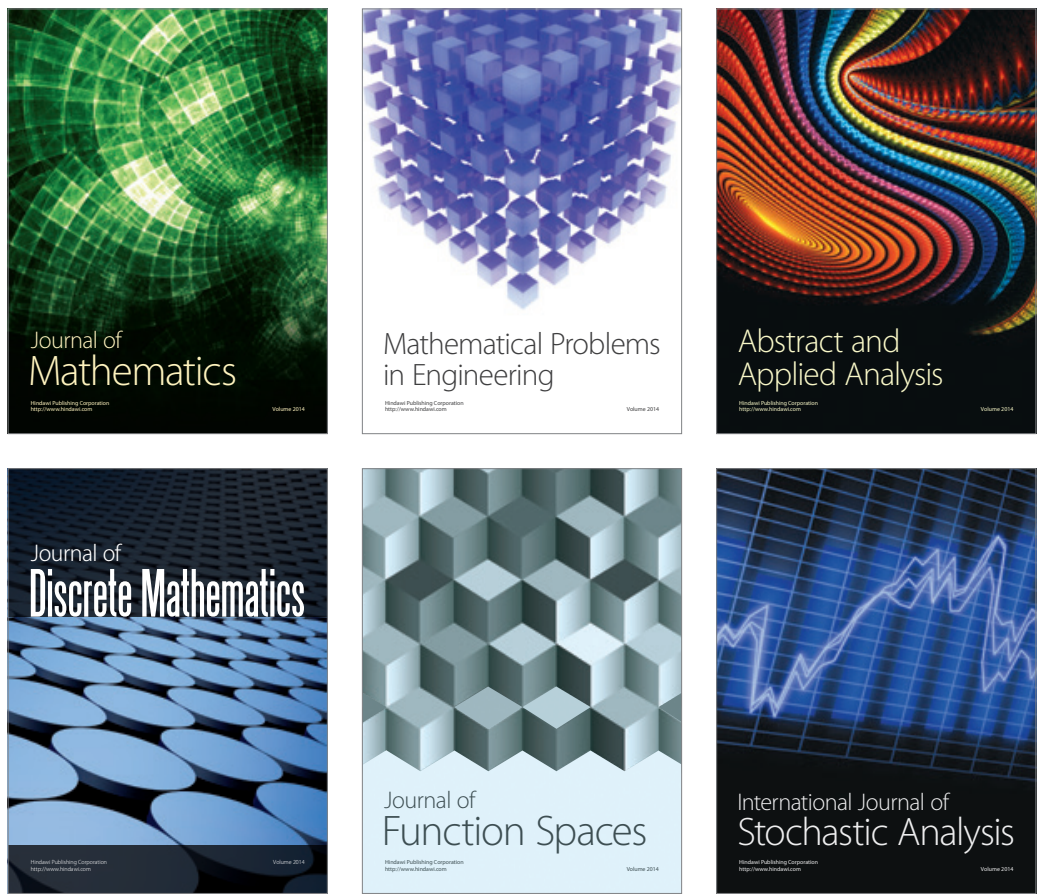

Journal of

Function Spaces

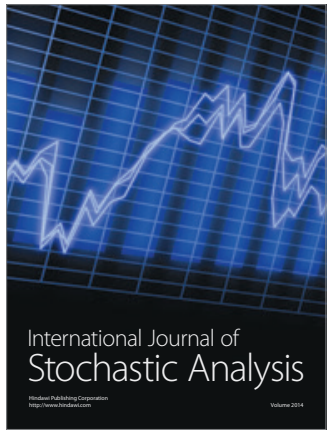

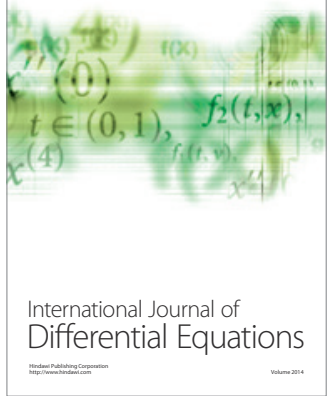
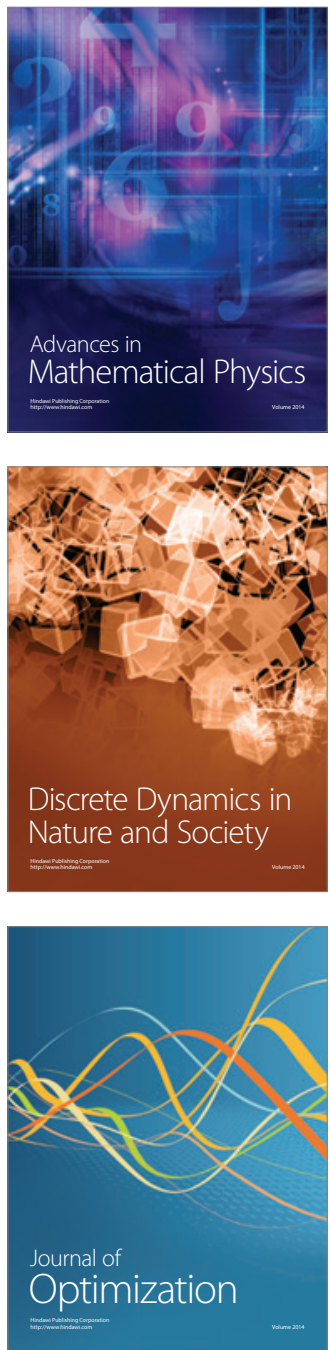